\title{
Double Virtual Compton Scattering and Spin Structure of the Nucleon
}

\author{
Hermann Krebs*† \\ Ruhr-Universität Bochum \\ Institut für Theoretische Physik II \\ E-mail: hermann.krebs@rub.de
}

\begin{abstract}
The internal spin structure of the nucleon is described by structure functions in deep inelastic lepton-hadron scattering which are related to real, virtual and double virtual Compton scattering amplitudes by various sum rules connecting information at all energy scales. In this proceeding I concentrate on theoretical description of spin polarizabilities of double virtual Compton scattering. We use chiral perturbation theory to describe low energy QCD dynamics in a model independent way. I briefly review chiral perturbation theory activities in this field and show that the Jefferson Lab experimental data at low virtuality are in fair agreement with ChPT calculations with explicit delta-resonance degrees of freedom. Though, preliminary experimental data indicate a disagreement with existing ChPT predictions. There are also discrepancies between two different calculations of generalized polarizabilities which use different power counting for delta degrees of freedom which will be discussed.
\end{abstract}

The 9th International workshop on Chiral Dynamics

17-21 September 2018

Durham, NC, USA

\footnotetext{
${ }^{*}$ Speaker.

$\dagger$ I would like to express my thanks to my collaborators Evgeny Epelbaum, Ulf-G. Meißner and Veronique Bernard for sharing their insight on the discussed topics. I also thank the organizers for the invitation and for making this exciting workshop possible. This work was supported in part by the DFG (SFB/TR 110, "Symmetries and the Emergence of Structure in QCD”) and the BMBF (Grant No. 05P18PCFP1).
} 


\section{Introduction}

Chiral perturbation theory (ChPT) is an established tool for analysis of low energy phenomena where the energy region is well below the chiral symmetry breaking scale $\Lambda_{\chi} \sim 1 \mathrm{GeV}$. Based on the approximate chiral symmetry of QCD, ChPT gives a systematic low energy expansion of QCD Green functions in mesonic sector [1]. Low energy meson sector has been studied within ChPT up to the order $p^{6}$ in chiral expansion which requires two-loop calculations [2]. Chiral Lagrangian is even worked out up to order $p^{8}$ [3] providing a path for systematic studies up to three-loops. An extension of ChPT to one nucleon sector is straightforward and is formulated as a general pathintegral in the presence of matter (nucleon) field [4]. In the last two decades low energy processes in the single nucleon sector have been intensively studied within ChPT, see [5]. Most of these studies correspond to full one-loop calculations in the delta-less and leading one-loop in a ChPT with explicit delta-resonance degrees of freedom (dof).

In this proceeding I report on our ChPT calculation of double virtual forward Compton scattering $\left(\mathrm{V}^{2} \mathrm{CS}\right)$ at low virtualities with explicit delta-resonance dof [6]. This study allows an indirect connection to experimental data by using sum rules. The sum rules provide a bridge between spinobservables of inclusive electroproduction and $\mathrm{V}^{2} \mathrm{CS}$. To be specific I will start with the inclusive electroproduction on one nucleon. Its differential cross section [7] is given by

$$
\frac{d^{2} \sigma}{d \Omega d E^{\prime}}=\frac{\alpha_{\mathrm{QED}}^{2}}{2 m_{N} q^{4}} \frac{E^{\prime}}{E} L_{\mu v} W^{\mu v}
$$

where $\alpha_{\mathrm{QED}}$ is a fine-structure constant, $m_{N}$ denotes the nucleon mass, $q$ is a virtual photon transfer momentum, $E$ and $E^{\prime}$ are initial and final electron energies, respectively. $L_{\mu \nu}$ and $W_{\mu \nu}$ denote leptonic and hadronic tensors. Hadronic tensor can be decomposed in the symmetric and antisymmetric parts

$$
W_{\mu v}=W_{\mu \nu}^{(S)}+W_{\mu \nu}^{(A)}
$$

which are denoted by labels " $S$ " and " $A$ ", and are relevant to unpolarized and polarized deep inelastic scattering, respectively. The antisymmetric hadron-tensor is a spin-dependent quantity and can be described by two structure functions $g_{1}$ and $g_{2}$

$$
W_{\mu \nu}^{(A)}=\frac{2 m_{N}}{P \cdot q} \varepsilon_{\mu \nu \alpha \beta} q^{\alpha}\left[S^{\beta} g_{1}\left(x, Q^{2}\right)+\left(S^{\beta}-\frac{S \cdot q}{P \cdot q} P^{\beta}\right) g_{2}\left(x, Q^{2}\right)\right],
$$

where $S$ is a spin-four-vector and

$$
x=\frac{Q^{2}}{2 P \cdot q}, \quad Q^{2}=-q^{2}
$$

Available polarized beams allow nowadays to study the structure functions $g_{1}\left(x, Q^{2}\right)$ and $g_{2}\left(x, Q^{2}\right)$. On the other hand various sum rules build a bridge between these observables and $\mathrm{V}^{2} \mathrm{CS}$ connecting information at all energy scales. It is one of the main goals of JLab activities to provide experimental mapping of spin-dependent observables from low-momentum to multi-GeV transfer. 
The spin-dependent part of $\mathrm{V}^{2} \mathrm{CS}$ in a forward kinematics can be described by two scalar functions $S_{1}$ and $S_{2}$ via

$$
\begin{aligned}
& \left.\left.i \int d^{4} x e^{i q \cdot x}\right\rangle P S\left|T J^{\mu}(x) J^{v}(0)\right| P S\right\rangle \\
= & -\frac{i}{2} \varepsilon^{\mu v \alpha \beta} q_{\alpha}\left[S_{\beta} S_{1}\left(v, Q^{2}\right)+\frac{1}{m_{N}^{2}}\left(P \cdot q S_{\beta}-S \cdot q P_{\beta}\right) S_{2}\left(v, Q^{2}\right)\right],
\end{aligned}
$$

where $J^{\mu}$ is the electromagnetic current,

$$
v=\frac{P \cdot q}{m_{N}}
$$

denotes photon energy, and $P$ and $S$ denote the momentum and spin polarization of the nucleon, respectively. After a subtraction of the elastic part, pole-part of the amplitude at $v=Q^{2} / 2 m_{N}$,

$$
\bar{S}\left(v, Q^{2}\right)=S\left(v, Q^{2}\right)-S^{\text {elastic }}\left(v, Q^{2}\right),
$$

one can build generalized polarizabilities defined by

$$
\begin{aligned}
\gamma_{0}\left(Q^{2}\right) & =\frac{1}{8 \pi}\left(\bar{S}_{1}^{(2)}\left(0, Q^{2}\right)-\frac{Q^{2}}{m_{N}} \bar{S}_{2}^{(3)}\left(0, Q^{2}\right)\right), \\
\delta_{L T}\left(Q^{2}\right) & =\frac{1}{8 \pi}\left(\bar{S}_{1}^{(2)}\left(0, Q^{2}\right)+\frac{1}{m_{N}} \bar{S}_{2}^{(1)}\left(0, Q^{2}\right)\right),
\end{aligned}
$$

where the moments are defined by

$$
\begin{aligned}
& \bar{S}_{1}\left(v, Q^{2}\right)=\sum_{i=0}^{\infty} \bar{S}_{1}^{(2 i)}\left(0, Q^{2}\right) v^{2 i} \\
& \bar{S}_{2}\left(v, Q^{2}\right)=\sum_{i=0}^{\infty} \bar{S}_{2}^{(2 i+1)}\left(0, Q^{2}\right) v^{2 i+1} .
\end{aligned}
$$

For low virtualities the moments $\bar{S}_{j}^{(k)}\left(0, Q^{2}\right)$ can be calculated within ChPT. On the other hand the generalized polarizabilities $\gamma_{0}\left(Q^{2}\right)$ and $\delta_{L T}\left(Q^{2}\right)$ can be obtained from sum rules such that ChPT predictions for $\gamma_{0}$ and $\delta_{L T}$ can be tested by experimental data or more precisely by integrals over data in the sum rules.

\section{ChPT with explicit delta-resonance}

In the original ChPT in the SU(2)-sector only pions and nucleons appear as dynamical dof. All resonances like delta- and higher-resonances are integrated out. Their contributions are explicitly accounted for in the low energy constants (LECs). From the studies of pion-nucleon scattering it is well known that LECs from second order pion-nucleon-Lagrangian are unnaturally large. The reason for this can be understood from delta-resonance saturation. Due to strong pion-nucleondelta coupling $h_{A} \sim 1.4$ and smallness of nucleon-delta-mass difference the resonance saturation contribution of the delta-resonance to LECs $c_{3}$ and $c_{4}$ which contribute to pion-nucleon scattering is unnaturally large. On top of this the leading non-minimal photon-nucleon-delta coupling which 
can be extracted from electromagnetic width of the delta-resonance appears also to be unnaturally large. In order to get a better convergence in ChPT calculation LECs should be of natural size. To enforce this it is advantageous to introduce delta-resonance as an explicit dof in a $\mathrm{V}^{2} \mathrm{CS}$ calculation. In our analysis [6] we used ChPT with explicit delta dof. ChPT with explicit deltas was introduced by Jenkins and Manohar [8], see also [9, 10] for early applications on decays of the baryon decuplet and electromagnetic polarizabilities of the nucleon. Later a matured version of ChPT with explicit deltas was introduced by Hemmert et al. [11]. In that work the authors proposed to treat momenta $Q$, pion mass $M_{\pi}$ and delta-nucleon mass difference $\Delta=m_{\Delta}-m_{N}$ phenomenologically as one universal small scale

$$
Q \sim M_{\pi} \sim \Delta \ll \Lambda_{\chi}
$$

denoted by $\varepsilon$. This scheme is known as the small scale expansion (SSE), also as the $\varepsilon$-power counting scheme. SSE is advantageous since it is consistent with the large $N_{c}$-expansion where in the $N_{c} \rightarrow \infty$ limit nucleons and delta dof are degenerate and should be treated in the same way. However, see [12] for a different so called $\delta$-power counting. However, in particular due to its simplicity, it is widely used in single nucleon calculations [13].

\section{3. $\mathbf{V}^{2} \mathrm{CS}$ up to $\varepsilon^{3}$ at photon point $Q^{2}=0$}

In our work [6] we used ChPT with explicit delta-resonance and performed SSE calculation up to $\varepsilon^{3}$. The work [6] was a natural extension of delta-less ChPT analyses [14, 15]. It is important to note that up to $\varepsilon^{3}$-order no unknown LECs appear. Chiral symmetry and gauge invariance restrict the form of $\mathrm{V}^{2} \mathrm{CS}$-amplitude allowing for the first spin-dependent two-photon-nucleon LEC to appear at order $\varepsilon^{5}$. This makes our calculation basically a prediction. Chiral Lagrangian and diagrams which contribute to $\mathrm{V}^{2} \mathrm{CS}$ up to $\varepsilon^{3}$ are explicitly given in [6]. For further discussion we give numerical values of LECs which contribute to $\mathrm{V}^{2} \mathrm{CS}$ :

$$
\begin{aligned}
g_{A} & =1.27, \quad F_{\pi}=92.21 \mathrm{MeV}, \quad M_{\pi}=138.04 \mathrm{MeV}, \quad m_{N}=938.9 \mathrm{MeV}, \\
\kappa_{v} & =3.706, \quad \kappa_{s}=-0.120, \quad m_{\Delta}=1232 \mathrm{MeV},
\end{aligned}
$$

denoting the axial nucleon coupling, pion decay constant, pion mass, nucleon mass, anomalous magnetic moments and delta-resonance mass, respectively. For delta-resonance couplings we use

$$
h_{A}=1.43 \pm 0.02, \quad b_{1}=-(4.98 \pm 0.27) / m_{N},
$$

where $h_{A}$ denotes pion-nucleon-delta and $b_{1}$ photon-nucleon-delta couplings at order $\varepsilon^{2}$, respectively. The values of the constants $h_{A}$ and $b_{1}$ in Eq. (3.2) have been determined from the strong and electromagnetic width of the delta-resonance. The values of these couplings are consistent with large- $N_{c}$ predictions given by

$$
h_{A}=\frac{3 g_{A}}{2 \sqrt{2}}=1.35, \quad b_{1}=-\frac{3}{2 \sqrt{2} m_{N}}\left(1+\kappa_{p}-\kappa_{n}\right)=-5.0 \frac{1}{m_{N}} .
$$

We start with the discussion of generalized polarizabilities $\gamma_{0}$ and $\delta_{L T}$ at the photon point. It is important to note that the first contribution to these observables start at order $\varepsilon^{3}$ such that we 
are talking about leading order predictions. The results at the photon point for central values of the input parameters are given by

$$
\begin{aligned}
& \gamma_{0}^{p}=2.07_{q^{3}}-3.65_{\mathcal{E}^{3}, \text { tree }}-0.16_{\mathcal{\varepsilon}^{3}, \text { loop }}=-1.74[ \pm 0.40], \\
& \gamma_{0}^{n}=3.06_{q^{3}}-3.65_{\varepsilon^{3}, \text { tree }}-0.18_{\mathcal{\varepsilon}^{3}, \text { loop }}=-0.77[ \pm 0.40] \text {, } \\
& \delta_{L T}^{p}=1.54_{q^{3}}-0.36_{\mathcal{E}^{3}, \text { tree }}+1.22_{\mathcal{E}^{3}, \text { loop }}=2.40[ \pm 0.01] \text {, } \\
& \delta_{L T}^{n}=2.41_{q^{3}}-0.36_{\mathcal{E}^{3}, \text { tree }}+0.33_{\mathcal{E}^{3}, \text { loop }}=2.38[ \pm 0.03] \text {. }
\end{aligned}
$$

All values are given in units of $10^{-4} \mathrm{fm}^{4}$. The first number in Eq. (3.4) labeled as " $q^{3 "}$ denotes the order- $q^{3}$ pion-nucleon contributions. The second term labeled by " $\varepsilon^{3}$, tree" denotes the order$\varepsilon^{3}$ tree-level contributions with one delta-resonance propagator. The third contribution labeled by $" \varepsilon^{3}$, loop" denotes the order- $\varepsilon^{3}$ one-loop contributions with at least one delta-resonance propagating in the loop integral. In brackets, we give the results due to the variation of $h_{A}$ and $b_{1}$ within the bounds given above. They should not be understood as an error estimate stemming from higher order truncations. From Eq. (3.4) we see that large positive contributions to generalized polarizabilities come from pion-nucleon loop diagrams. Delta-resonance contributions behave differently for $\gamma_{0}$ and $\delta_{L T}$. In the case of $\gamma_{0}$ one gets large but negative contributions from $\varepsilon^{3}$ tree-level diagrams and small contributions from $\varepsilon^{3}$ loop diagrams with delta dofs. There appear strong cancelations between pion-nucleon loop and $\varepsilon^{3}$ delta-resonance tree-level contributions such that $\gamma_{0}$ becomes a fine-tuned quantity. For this reason one can not expect a precise description of $\gamma_{0}$ at the order $\varepsilon^{3}$ in the SSE. In the case of $\delta_{L T}$ we see an opposite qualitative behavior: $\varepsilon^{3}$ tree level diagrams with delta-resonance give small contribution while $\varepsilon^{3}$ one-loop diagrams with delta dofs seem to be sizeable, at least for proton case. There is no cancelation between pion-nucleon loops and deltaresonance contributions which makes $\delta_{L T}$ a preferable quantity for a test of ChPT. Though, a size of $\varepsilon^{3}$-order one-loop contributions with delta dofs to $\delta_{L T}$ is surprising since it seems to contradict a general consideration based on multipole decomposition of pion-electroproduction from which one expects that delta-resonance contributions to $\delta_{L T}$ should be suppressed [16]. To clarify this issue let us briefly discuss the arguments of [16]. According to Drechsel et al. [16] $\delta_{L T}$ and $\gamma_{0}$ can be constructed via dispersion integral from pion electroproduction amplitudes, see Fig 1. According to Eqs (25) and (26) of [16] $\gamma_{0}$ and $\delta_{L T}$ can be expressed in terms of pion-electroproduction multipole amplitudes

$$
\begin{aligned}
\gamma_{0} & =\frac{2}{\pi} \int \frac{d v}{v^{3}} \frac{k_{\pi}^{c m}}{v} \sqrt{1+\frac{2 v}{m_{N}}}\left(\left|E_{0+}\right|^{2}-\left|M_{1+}\right|^{2}+6 E_{1+}^{*} M_{1+}+3\left|E_{1+}\right|^{2}+\ldots\right), \\
\delta_{L T} & =\frac{2}{\pi} \int \frac{d v}{v^{3}} \frac{k_{\pi}^{c m}}{v}\left(1+\frac{2 v}{m_{N}}\right)\left(L_{0+}^{*} E_{0+}+2 L_{1+}^{*}\left(M_{1+}+3 E_{1+}\right)+\ldots\right)
\end{aligned}
$$

with

$$
k_{\pi}^{c m}=\frac{1}{2 W} \sqrt{\left(W^{2}-M_{\pi}^{2}\right)^{2}-2 m_{N}^{2}\left(W^{2}+M_{\pi}^{2}\right)+m_{N}^{4}}, \quad W=\sqrt{m_{N}^{2}+2 m_{N} v-Q^{2}} .
$$

Due to the $v^{-3}$ term in the integrands high- $v$ modes are strongly suppressed such that multipole expansion of pion-electroproduction can be expected to converge rapidly. As a consequence a term proportional to $\left|E_{0+}\right|^{2}$ in the case of $\gamma_{0}$ is enhanced and nearly cancels contributions from magnetic 


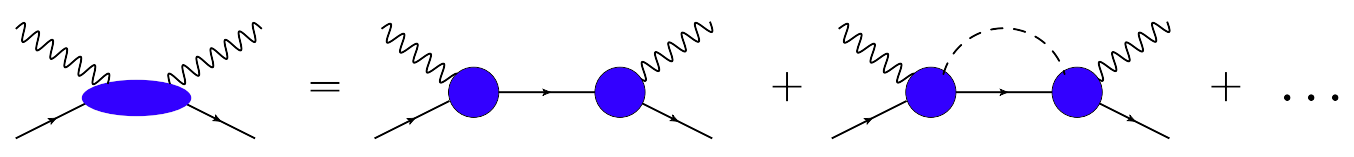

Figure 1: Forward spin polarizabilities via dispersion integral of pion electroproduction amplitudes. Elastic part and pion-nucleon cut contribution are sketched. Two and more-pion-nucleon cuts are dropped and denoted by dots.

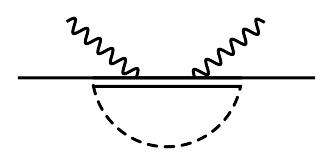

Figure 2: Box diagram which gives a sizeable contribution to $\delta_{L T}$ polarizability.

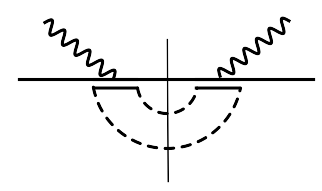

Figure 3: Box diagram of Fig. 2 represented as dispersion integral with two-pion-nucleon cut.

delta-resonance excitation proportional to $\left|M_{1+}\right|^{2}$. Due to this cancelation the interference term proportional to $E_{1+}^{*} M_{1+}$ becomes important. In the case of $\delta_{L T}$ there is no such cancelation such that $\delta_{L T}$ is dominated by s-wave terms proportional to $L_{0+}^{*} E_{0+}$. The contributions from magnetic delta-resonance excitation proportional to $L_{1+}^{*} M_{1+}$ is therefore expected to be suppressed. This expectation seems to be in contradiction with Eq. (3.4) where we see large contribution from delta resonance dofs: Delta tree-level contributions to $\delta_{L T}$ are indeed small. However loop-contributions with delta-resonances are sizeable. In order to clarify where this effect comes from we analyzed loop-diagrams with delta-resonance involved and have seen in the gauge $\varepsilon_{0}=0$, where $\varepsilon_{\mu}$ denotes virtual photon polarization four-vector, that the main contribution to $\delta_{L T}$ comes from the boxdiagram from Fig. 2. At the photon point the sum of this and crossed-box diagram gives

$$
\delta_{L T}^{p}(\mathrm{box})=1.32 \times 10^{-4} \mathrm{fm}^{4} .
$$

To show that there is no contradictions with arguments in [16] we note that delta-resonance is not an asymptotic state. Only nucleons and pions are asymptotic states in ChPT. Within dispersionintegral the box diagram of Fig. 2 corresponds to two-pion-exchange contributions with twopion-nucleon cut, see Fig. 3. Two-pion-electroproduction amplitude was not taken into account in [16] but assumed to give a small contribution due to large phase space suppression. In the explicit calculation we see, however, a sizeable contribution from the box diagram. To exclude the possibility that the large effects are due to an inappropriate renormalization scheme we plot in Fig. 4 the box-diagram contribution to $\delta_{L T}^{p}$ in dependence on delta-resonance mass. From Fig. 4 we see that delta-resonance indeed decouples for larger values of the delta-resonance mass showing the proper ultraviolet behavior of the box diagram. Enhanced contribution of the box diagram in 


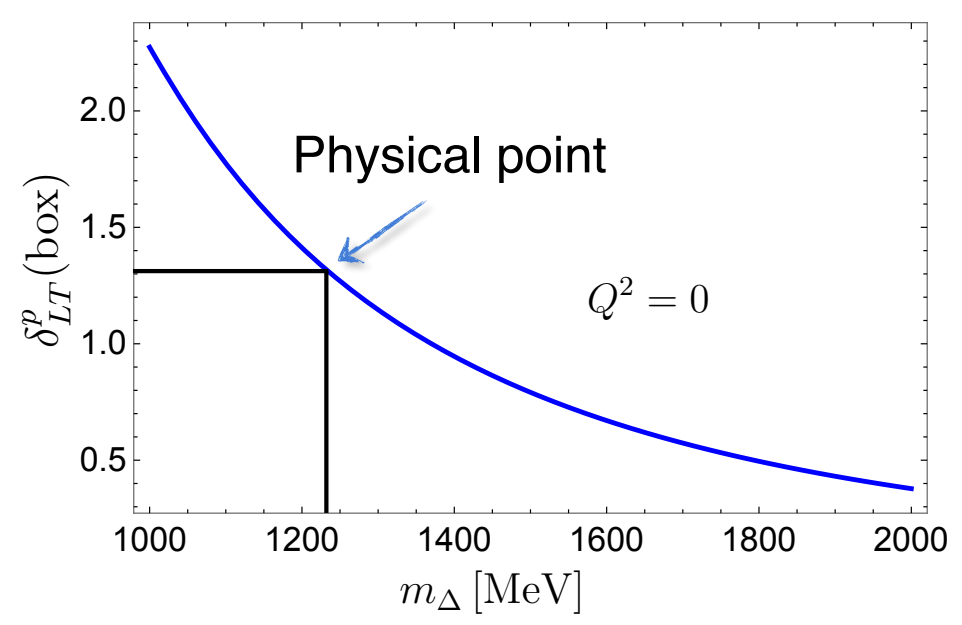

Figure 4: The contribution of the box diagram to $\delta_{L T}^{p}$ in dependence of delta-resonance mass $m_{\Delta}$ at photon point $Q^{2}=0$.

the proton case can be understood from its isospin prefactor given by

$$
\delta_{L T}(\text { box })=\left(9+5 \tau_{3}\right) f_{L T},
$$

where $\tau_{3}$ is a Pauli matrix and $f_{L T}$ does not depend on isospin dofs. Eq. (3.8) indicates roughly a factor three enhancement for proton compared to neutron contributions:

$$
\delta_{L T}^{p}(\text { box })=14 f_{L T}, \quad \delta_{L T}^{n}(\text { box })=4 f_{L T} .
$$

\section{4. $\mathrm{V}^{2} \mathrm{CS}$ up to $\varepsilon^{3}$ at finite virtuality}

After an extensive discussion of generalized polarizabilities $\gamma_{0}$ and $\delta_{L T}$ at the photon point $Q^{2}=0$ we come to the results at finite virtuality. In Fig. 5 we show generalized polarizabilities $\gamma_{0}$ and $\delta_{L T}$ in dependence on the virtuality $Q^{2}$. Red bands are our predictions from [6]. The bands do not include systematic uncertainties but indicate only the sensitivity to the variation of $h_{A}$ and $b_{1}$ as given in Eq. (3.2). Blue bands are ChPT predictions in the $\delta$-counting [18] which use a somewhat different value for $b_{1}$ given by

$$
b_{1}=3 g_{M} /\left(m_{N}+m_{\Delta}\right)=3.85 / m_{N}
$$

corresponding to $g_{M}=2.97$ from [17]. In order to compare our results with those of [17] we give in Fig. 5 our predictions for $b_{1}=3.85 / m_{N}$ (red curve). As can be seen from Fig. 5 both calculations give qualitatively similar results on $\gamma_{0}$ and $\delta_{L T}^{n}$. However, for $\delta_{L T}^{p}$ the results differ, in particular due to the absence of the box-diagram in $\delta$-counting. The box diagram contributes in the $\delta$-counting at higher than $p^{4} / \Delta$-order. It is important to note that all diagrams which appear in the $\delta$-counting at order $p^{4} / \Delta$ appear also in SSE at order $\varepsilon^{3}$. In SSE at order $\varepsilon^{3}$ there are, 

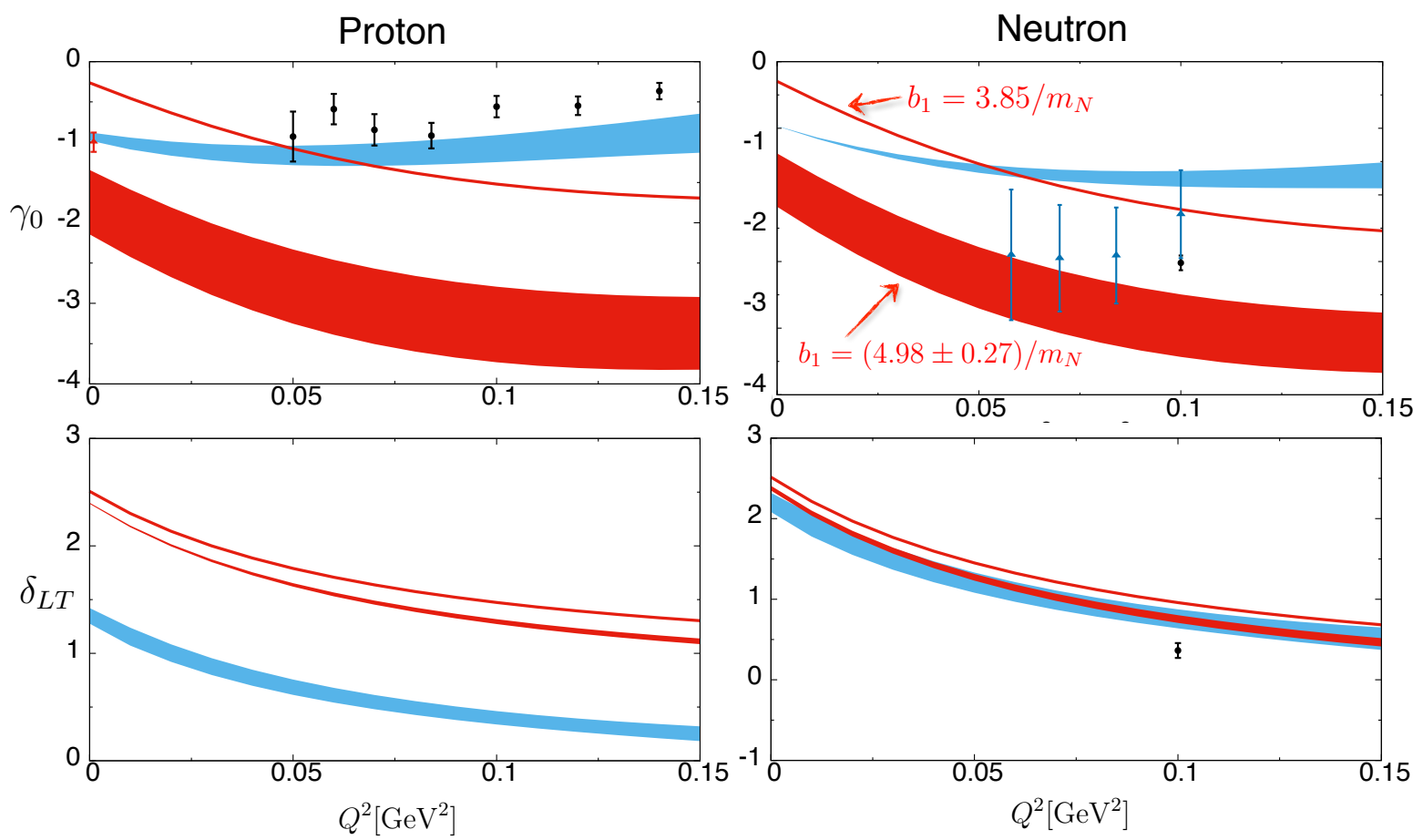

Figure 5: Generalized polarizabilities $\gamma_{0}$ and $\delta_{L T}$ in dependence on virtuality $Q^{2}$. Red lower bands are SSE predictions at order $\varepsilon^{3}$ which indicate the sensitivity to LECs $h_{A}$ and $b_{1}$ which was varied as given in Eq. (3.2). For comparison reason we also give SSE predictions at order $\varepsilon^{3}$ for $b_{1}=3 g_{M} /\left(m_{N}+m_{\Delta}\right)=$ $3.85 / m_{N}$ (upper red curve) which corresponds to the value $g_{M}=2.97$ given in [17]. Blue bands are ChPT prediction in $\delta$-counting from [18]. Neutron data: Ref. [19] and proton data from Ref. [20] $\left(Q^{2}=0\right)$ and Ref. [21] $\left(Q^{2}>0\right)$. Only statistical errors are shown.

however, other diagrams with two and three delta-resonance propagators which are counted in $\delta$ counting as higher order effects. The actual smallness of these effects can be tested by comparing SSE and $\delta$-counting calculations. A strong disagreement on $\delta_{L T}^{p}$ indicates that higher order effects are sizeable. For this reason it is important to go in both calculations at least one order higher. Fig. 5 shows a fair agreement with experimental data in both calculations for the lower value of $b_{1}$, namely $b_{1}=3.85 / m_{N}$. However, preliminary experimental data [22] at lower virtuality seem to disagree with both calculations. This gives also a motivation to go one order higher both in SSE and $\delta$-counting scheme calculations.

\section{Summary and conclusions}

I discussed ChPT calculation of generalized polarizabilities $\gamma_{0}$ and $\delta_{L T}$ of double-virtual forward Compton scattering up to $\varepsilon^{3}$ in the small scale expansion [6]. First contributions to polarizabilities start at $\varepsilon^{3}$ so this is a leading order calculation. Gauge symmetry restricts the form of the chiral Lagrangian such that there is no spin-dependent low energy constant at order $\varepsilon^{3}$. For this reason there are no fitting parameters and we are talking here about a prediction. I demonstrated that our predictions for $\gamma_{0}$ and $\delta_{L T}$ are in fair agreement with experimental data. However, preliminary experimental data at lower virtuality [22] indicate a disagreement between our prediction and 
experimental data. Similar ChPT calculations of generalized polarizabilities have been performed within a $\delta$-counting scheme [18]. The diagrams which contribute in the $\delta$-counting scheme at the order $p^{4} / \Delta$ are also part of diagrams which contribute at order $\varepsilon^{3}$ in the small scale expansion. However, $\varepsilon^{3}$-order calculation includes more diagrams than those which appear at $p^{4} / \Delta$ order in the $\delta$-counting scheme. In particular, a box-diagram of Fig. 2 with three delta-resonance propagators contributes to $\varepsilon^{3}$-order calculation but is counted as higher order than $p^{4} / \Delta$ in $\delta$-counting scheme and has not been taken into account in [18]. In the $\varepsilon_{0}=0$ gauge this diagram gives a sizeable contribution to $\delta_{L T}^{p}$ and leads to strong disagreement between the $\varepsilon$ - and $\delta$-counting calculations. Also one should point out that the input Lagrangians in [18] and in [6] are different. The difference are in the off-shell spin-1/2 ambiguities of the delta-resonance. Up to the order we are working these differences are, however, negligible. Due to strong disagreement between two ChPT calculations one should go at least one order higher in $\varepsilon$ and $\delta$-counting scheme calculations.

\section{References}

[1] J. Gasser and H. Leutwyler, Annals Phys. 158, 142 (1984). doi:10.1016/0003-4916(84)90242-2

[2] J. Bijnens, Prog. Part. Nucl. Phys. 58, 521 (2007) doi:10.1016/j.ppnp.2006.08.002 [hep-ph/0604043].

[3] J. Bijnens, N. Hermansson-Truedsson and S. Wang, JHEP 1901, 102 (2019) doi:10.1007/JHEP01(2019)102 [arXiv:1810.06834 [hep-ph]].

[4] J. Gasser, M. E. Sainio and A. Svarc, Nucl. Phys. B 307, 779 (1988). doi:10.1016/0550-3213(88)90108-3

[5] V. Bernard, Prog. Part. Nucl. Phys. 60, 82 (2008) doi:10.1016/j.ppnp.2007.07.001 [arXiv:0706.0312 [hep-ph]].

[6] V. Bernard, E. Epelbaum, H. Krebs and U.-G. Meißner, Phys. Rev. D 87, no. 5, 054032 (2013) doi:10.1103/PhysRevD.87.054032 [arXiv:1209.2523 [hep-ph]].

[7] S. E. Kuhn, J.-P. Chen and E. Leader, Prog. Part. Nucl. Phys. 63, 1 (2009) doi:10.1016/j.ppnp.2009.02.001 [arXiv:0812.3535 [hep-ph]].

[8] E. E. Jenkins and A. V. Manohar, UCSD-PTH-91-30.

[9] M. N. Butler, M. J. Savage and R. P. Springer, Nucl. Phys. B 399, 69 (1993) doi:10.1016/0550-3213(93)90617-X [hep-ph/9211247].

[10] M. N. Butler and M. J. Savage, Phys. Lett. B 294, 369 (1992) doi:10.1016/0370-2693(92)91535-H [hep-ph/9209204].

[11] T. R. Hemmert, B. R. Holstein and J. Kambor, J. Phys. G 24, 1831 (1998) doi:10.1088/0954-3899/24/10/003 [hep-ph/9712496].

[12] V. Pascalutsa and D. R. Phillips, Phys. Rev. C 67, 055202 (2003) doi:10.1103/PhysRevC.67.055202 [nucl-th/0212024].

[13] F. Hagelstein, R. Miskimen and V. Pascalutsa, Prog. Part. Nucl. Phys. 88, 29 (2016) doi:10.1016/j.ppnp.2015.12.001 [arXiv:1512.03765 [nucl-th]].

[14] V. Bernard, T. R. Hemmert and U.-G. Meißner, Phys. Rev. D 67, 076008 (2003) doi:10.1103/PhysRevD.67.076008 [hep-ph/0212033]. 
[15] V. Bernard, T. R. Hemmert and U.-G. Meißner, Phys. Lett. B 545, 105 (2002) doi:10.1016/S0370-2693(02)02584-4 [hep-ph/0203167].

[16] D. Drechsel, S. S. Kamalov and L. Tiator, Phys. Rev. D 63, 114010 (2001) doi:10.1103/PhysRevD.63.114010 [hep-ph/0008306].

[17] V. Pascalutsa, M. Vanderhaeghen and S. N. Yang, Phys. Rept. 437, 125 (2007) doi:10.1016/j.physrep.2006.09.006 [hep-ph/0609004].

[18] V. Lensky, J. M. Alarcón and V. Pascalutsa, Phys. Rev. C 90, no. 5, 055202 (2014) doi:10.1103/PhysRevC.90.055202 [arXiv:1407.2574 [hep-ph]].

[19] M. Amarian et al. [Jefferson Lab E94010 Collaboration], Phys. Rev. Lett. 93, 152301 (2004) doi:10.1103/PhysRevLett.93.152301 [nucl-ex/0406005].

[20] H. Dutz et al. [GDH Collaboration], Phys. Rev. Lett. 91, 192001 (2003). doi:10.1103/PhysRevLett.91.192001

[21] Y. Prok et al. [CLAS Collaboration], Phys. Lett. B 672, 12 (2009) doi:10.1016/j.physletb.2008.12.063 [arXiv:0802.2232 [nucl-ex]].

[22] A. Deur, arXiv:1903.05661 [nucl-ex]. 